\title{
Delayed disengagement from irrelevant fixation items: Is it generally functional?
}

\author{
Maximilian Stefani $^{1}$ (D) $\cdot$ Marian Sauter $^{1} \cdot$ Wolfgang Mack $^{1}$ \\ Published online: 23 January 2020 \\ (C) The Psychonomic Society, Inc. 2020
}

\begin{abstract}
In a circular visual search paradigm, the disengagement of attention is automatically delayed when a fixated but irrelevant center item shares features of the target item. Additionally, if mismatching letters are presented on these items, response times (RTs) are slowed further, while matching letters evoke faster responses (Wright, Boot, \& Brockmole, 2015a). This is interpreted as a functional reason of the delayed disengagement effect in terms of deeper processing of the fixation item. The purpose of the present study was the generalization of these findings to unfamiliar symbols and to linear instead of circular layouts. Experiments 1 and 2 replicated the functional delayed disengagement effect with letters and symbols. In Experiment 3, the search layout was changed from circular to linear and only saccades from left to right had to be performed. We did not find supportive data for the proposed functional nature of the effect. In Experiments 4 and 5, we tested whether the unidirectional saccade decision, a potential blurring by adjacent items, or a lack of statistical power was the cause of the diminished effects in Experiment 3 . With increased sample sizes, the delayed disengagement effect as well as its functional underpinning were now observed consistently. Taken together, our results support prior assumptions that delayed disengagement effects are functionally rooted in a deeper processing of the fixation items. They also generalize to unfamiliar symbols and linear display layouts.
\end{abstract}

Keywords Attentional capture $\cdot$ Attentional disengagement $\cdot$ Visual search $\cdot$ Contingent capture $\cdot$ Attention

\section{Introduction}

Imagine that you are driving your car on the highway and you notice a traffic sign that you have never seen before. This sign captures your attention and you will keep looking at it to figure out what it means. Now how does your visual system determine such an attentional dwell time on specific objects in the visual scene? In a standard visual search task, attention can be captured by irrelevant items that are the most salient objects in the search display (Theeuwes, 1994, 2004). This influence is described as bottom-up or stimulus-driven capture. In addition, attention can be captured by items that are irrelevant but have some prominent features in common with the search

Maximilian Stefani and Marian Sauter contributed equally to the work.

Maximilian Stefani

maximilian.stefani@unibw.de

$1 \quad$ Institute of Psychology, General Psychology, Bundeswehr University Munich, Werner-Heisenberg-Weg 39, 85577 Neubiberg, Germany target (Folk, Remington, \& Johnston, 1992; Leber \& Egeth, 2006), a process typically referred to as top-down or goal-directed.

Irrelevant but target-similar items also attract attention even when they occur at a place where the target item cannot appear, an effect referred to as contingent capture (Folk et al., 1992; Folk, Leber, \& Egeth, 2002). In a classical contingent capture study (Folk et al., 2002), observers had to identify a target object within a rapid serial visual presentation stream. On some trials, irrelevant color singletons (distractors) appeared in the periphery. The authors showed that target identification decreased if the distractors had the same color as the target. In this case, attention briefly shifted to the irrelevant peripheral objects. Brockmole and Boot (2009) note that such contingent capture studies typically only investigated the pull of attention towards irrelevant items in the periphery. They reason that not only pulling attention towards peripheral objects but also holding attention at specific objects is an important determinant of visual processing. In their study, they were able to show that color singletons not only captured attention when presented in the periphery, but also held attention (i.e., delayed the disengagement) when presented at fixation. 
Critically, fixation items only delayed attention when they were unique (singletons) and unexpected (novel color) at the same time, that is, bottom-up salience alone was not sufficient to hold attention at fixation (cf. Born, Kerzel, \& Theeuwes, $2011 \mathrm{~b}$, who found that dwell time was driven by targetdistractor similarities). Therefore, they attribute top-down factors as being the major determinant of the delayed disengagement effect. In another delayed disengagement study (Boot \& Brockmole, 2010), observers were requested to saccade away from an always irrelevant center object to a target-colored object out of several objects that were presented in a circular arrangement. The participants had to decide which of two possible letters was contained in the target object. In this circular layout, the center object also served as a distractor. If the distractor color matched the target color (i.e., is related to the observer's task goal) or was presented unexpectedly, saccadic latency increased significantly (Boot \& Brockmole, 2010; Brockmole \& Boot, 2009). These findings indicate a consistent delay in disengagement depending on the attentional set of the observer even if the irrelevant object does not exactly match the search target's features (Blakely, Wright, Dehili, Boot, \& Brockmole, 2012; Wright, Boot, \& Jones, 2015b), and suggest that top-down factors are critical to the delay.

Wright, Boot, and Brockmole (2015a) suggested that these delays serve a functional purpose. They proposed two alternative explanations of delayed disengagement effects. It could be either because of "a need to allocate additional time to locating the peripheral target or to filtering/inhibiting the fixated item" or because it encourages deeper levels of processing. In their delayed disengagement study, the authors found a letter congruency effect but only for target-similar distractors at fixation: When attention is captured by a distractor that has features similar to the attentional set, the observer processes the distractor and its features at a deeper level. More precisely, they hypothesized that only if an irrelevant but fixated object shares the same color with the target object, the inner letters from distractor and target object are processed. Matching inner letters in target and target-similar distractors are processed faster than non-matching inner letters (Wright, Boot, \& Brockmole, 2015a). They conclude that through the engagement of top-down control, items that share features with the target object in the attentional set could delay disengagement because such items "receive more scrutiny" and are processed more deeply. This deeper processing of target-similar items is thought to aid in identifying relevant target objects (Belopolsky, Schreij, \& Theeuwes, 2010; Wright, Boot, \& Jones, 2015b) and prevent premature disengagement from "candidate" target objects. Delaying the disengagement from target "candidates" can only facilitate search performance if such a delay is accompanied by a deeper (more detailed) processing of the attended item. Therefore, investigating the (functional) reason for the delayed disengagement can provide insight into mechanisms of visual processing during visual search in distracting environments. Critically, it was recently found that the attentional dwell time at a distractor location scales with search difficulty and is an important determinant of the widely discussed attentional capture effects (Gaspelin, Ruthruff, \& Lien, 2016). This frames the delayed disengagement effect as directly related to attentional capture effects. Accordingly, investigating the functional reason for the delayed disengagement effect seems to promise a deepening of our understanding of attentional capture mechanisms.

Because the Wright, Boot, and Brockmole (2015a) study remains the only study that described delayed disengagement as functional in terms of deeper processing of the fixation item, we remained unconvinced that a deeper level of processing caused the delay in disengagement from the center circle because of their choice of stimuli. Using letters as stimuli for response decisions might not be ideal because they are especially familiar to the observers. Treisman and Gelade (1980) already stated that "letters have long been controversial units in perceptual theory." They might even be automatically processed as a whole without attentional effort (Laberge, 1973) and further automatically recruit a sensory-motor brain network (James \& Gauthier, 2006), which might subserve automatized response processes. To get an untainted picture of the hypothesized - general functional nature of the delayed disengagement effect (i.e., deeper processing of fixation items), it seems reasonable to use unfamiliar symbols that are less automatically processed than letters. In Experiment 1, we replicated Wright, Boot, and Brockmole (2015a) without eye tracking. Experiment 2 was intended to reveal similar color congruency effect and letter congruency effects as in Experiment 1 and in Wright, Boot, and Brockmole (2015a). However, in Experiment 2 we opted for unfamiliar star-like shapes with six or eight corners that were hard to discriminate (as ensured in a pre-test comparison of triangles, squares, and star-like shapes).

To the best of our knowledge, the delayed disengagement effect - and its hypothesized function - was reported exclusively in connection with using circular search displays. In such circular layouts, the search target can be in any direction from the center circle. However, in everyday life, we move our eyes most often horizontally. Vertical and oblique saccades are less accurate and generally slower (Becker \& Jürgens, 1990). Horizontal saccades are more "natural" and performed faster, in terms of both saccadic latency and peak velocity. It is therefore possible that the delayed disengagement effect appears the most robust (i.e., with less variance) in experimental designs that only incorporate horizontal eye movements. For this reason, a corresponding design was adapted in Experiment 3: All seven circles were now arranged in a straight line with the starting circle always being the left-most one. This means that to perform the task, only left-to-right-saccades have to be made. With such a linear search display we can additionally test whether delayed disengagement and functional delayed 
disengagement is dependent on unforeseen characteristics of the kind of layout configuration. Experiment 4 was set-up post hoc, because we did not find a general letter congruency effect in Experiment 3 (see below). Consequently, in Experiment 4, we built on Experiment 3 to differentiate between two possible explanations of the missing letter congruency effect in Experiment 3. Either the delay in disengagement was the result of deeper processing, but the letter congruency effect thought to be indicative of this - was too fragile and overwritten before the target was reached, or the delay in disengagement was a result of the layout configuration and is generally not accompanied by deeper processing of the fixation item in linear designs (for a detailed description, see discussion of Experiment 3 and introduction of Experiment 4).

\section{Experiment 1 - Letters}

Experiment 1 aimed to replicate the behavioral findings of Wright, Boot, and Brockmole (2015a). The major interest lay in successful replication of the letter congruency effect. This means that only if the center circle and target circle are of the same color, RTs are expected to be slower when the inner letters do not match (i.e., are incongruent).

\section{Methods}

Participants In Experiment 1, we replicated the study of Wright, Boot, and Brockmole (2015a) with 37 students (17 females and 19 males; $19-38$ years old, $M=24.3, S D=4.52$ ) of the Bundeswehr University Munich. All participants had normal or corrected-to-normal vision. They provided informed consent and were given course credit as compensation for their participation.

Setup The tasks were presented on an Asus LCD 144-Hz screen at a distance of $68 \mathrm{~cm}$. Button presses were recorded with the Black Box ToolKit USB response pad (The Black Box ToolKit Ltd). The visual search displays constituted of six gray circles (RGB: 196, 196, 196) that were positioned at a visual angle of $7.8^{\circ}$ around a center circle on a white background. Each circle (1.4 ${ }^{\circ}$ radius) contained a smaller gray circle $\left(.3^{\circ}\right.$ radius) except for the center circle, which contained both a gray circle $\left(.6^{\circ}\right.$ radius $)$ and a black-lined square $\left(.6^{\circ}\right.$ radius). In each trial, participants had to fixate the center circle presented for $500,1,000$, or $1,500 \mathrm{~ms}$, whereas presentation times were counterbalanced and distributed randomly. Immediately afterwards, all circles changed their color simultaneously. The center circle changed to green (RGB: 0, 255, 0 ), red (RGB: 255, 0, 0), or blue (RGB: 0, 0, 255), four of the six peripheral circles changed to yellow (RGB: 255, 255, 0), one to the target color (green or blue), and one to red. Target color was implemented as a between-subject condition counterbalanced across participants. At the same time as the color changes, letters (A, F, H, O, U, and target letter ' $\mathrm{C}$ ' or ' $\left.\mathrm{O}^{\prime} ; 0.4^{\circ} \times 0.4^{\circ}\right)$ appeared in the six peripheral circles and the square in the center circle changed to a ' $\mathrm{C}$ ' or a ' $\mathrm{J}$ ' (congruent or incongruent with the target letter). The color of the center circle and its inner letter predicted neither the location nor the letter of the target circle. Distractor location, target location, target letter identity (' $\mathrm{C}$ ' or ' $\mathrm{J}$ '), and center letter identity (' $\mathrm{C}$ ' or ' $\mathrm{J}$ ') were counterbalanced and presented in random order (see Fig. 1).

One difference between our study and that of Wright, Boot, and Brockmole (2015a) was that we did not employ eye tracking in this experiment. This implies that we had to adapt the calculation of the RTs. In the original study, (manual) RTs were measured from the time of the first fixation after an accurate saccade to the target circle until button press. Since this is not possible without eye tracking, we measured "classical" RTs from the time the search display was uncovered until button press. Also, in contrast to the original study, we did not mask the center letter after oculomotor disengagement from the fixation item.

Task The instruction was to look at the target-colored circle (green or blue) as quickly as possible and press one of the buttons to indicate the answer, left button for ' $\mathrm{C}$ ' and right button for ' $O$ ', respectively. The colors and letters of all other circles, even the center circle, were task irrelevant and were to be ignored. The participants completed 360 trials in total. The first 20 trials were for practice and were not included in analyses.

\section{Results}

We did not exclude any outliers based on response times. Accuracy did not interact with RT distributions, $F(2,68)=$ $1.46, p=.24, \eta^{2}=.04$, and error rate was below $1 \%$ in all conditions; therefore, we only analyzed correct trials. Further, we report median RTs per participant and condition. Calculations were done using jamovi 0.9 (jamovi project, 2018) .

To test the hypothesis that congruent colors lead to slower RTs compared to incongruent colors (color congruency effect), we first averaged over both congruent-center target and incongruent-center target color combination conditions. In the following, blue-blue and green-green combinations are referred to as congruent combinations, blue-green and green-blue as incongruent combinations, and red-green and red-blue as neutral combinations. ${ }^{1}$ We applied a similar logic

\footnotetext{
${ }^{1}$ We did this because in the Wright, Boot, and Brockmole (2015a) study, effect sizes were quite small and there were no significant differences between the afore-mentioned conditions, either in Wright, Boot, and Brockmole or in our raw RTs, $F(1,34)=1.72, p=.20, \eta^{2}=.048$.
} 


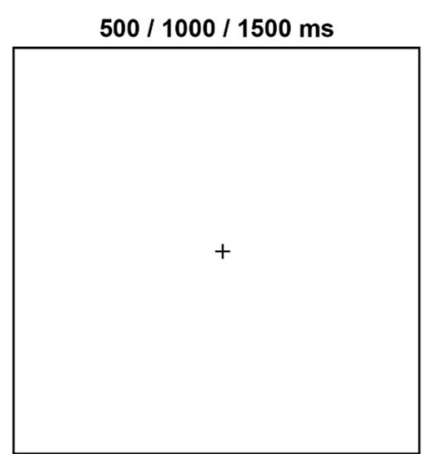

$500 / 1000 / 1500 \mathrm{~ms}$

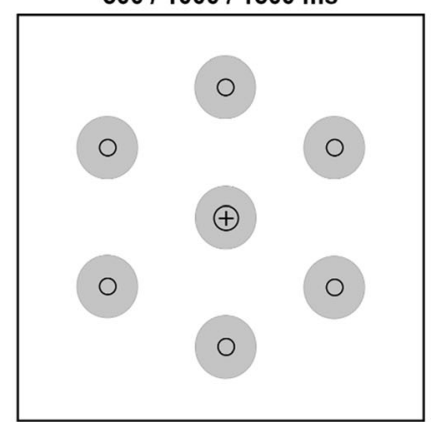

blue (or the green) peripheral circle and decide whether the target letter was a ' $C$ ' or ' $O$ '. All other features of the other circles and the center circle were to be ignored to different colors. Participants were instructed to always search for the

to letter congruency regarding combinations of the center and target circle: C-C-combinations and J-J combinations are referred to as congruent combinations, J-C or C-J combinations are referred to as incongruent combinations.

To test for a slowdown in RTs for congruent letter combinations as compared to incongruent letter combinations, a repeated-measures ANOVA was calculated with color congruency (neutral, incongruent, or congruent) and letter congruency (incongruent or congruent) as within-subject factors. The main effect for color congruency was highly significant, $F(2$, $72)=34.59, p<.001, \eta^{2}=.49$. The main effect for letter congruency was not significant, $F(1,36)=0.30, p=.059, \eta^{2}$ $=.008$. The interaction was significant, $F(2,72)=15.55, p<$ $.001, \eta^{2}=.30$. According to Boot and Brockmole (2010), the color congruency effect indicates oculomotor capture, which they interpreted as delayed disengagement. A slowdown for congruent color combinations (compared to incongruent or neutral combinations) is supposed to be a prerequisite for the functional delayed disengagement (in a sense of deeper processing of the fixation item), and only then is the letter congruency effect expected as observed in Wright, Boot, and Brockmole (2015a). Therefore, in post hoc tests, we contrasted the color combinations and revealed significant differences for neutral $(695 \mathrm{~ms})$ versus congruent $(730 \mathrm{~ms})$ color combinations, $t(72)=7.20, p<.001, d=0.57$ and incongruent $(696 \mathrm{~ms})$ versus congruent $(730 \mathrm{~ms})$ color combinations, $t(72)=7.21, p<.001, d=0.54$, but not for neutral (695 ms) versus incongruent (696 ms) color combinations, $t(72)=0.00, p=.99, d=0.018$ (see Fig. 2). These results support Boot and Brockmole's (2010) conclusion that only congruent color combinations delay responses.

Next, we analyzed the letter congruency effect. There was a significant slowdown in RTs for incongruent versus congruent letter combinations only for congruent color combinations, $M$ $=26 \mathrm{~ms}, t(102)=4.51, p<.001, d=0.36$ (see also Fig. 2 ), but not for incongruent color combinations, $M=11 \mathrm{~ms}, t(102)=$ $1.95, p=.054, d=0.19$, or neutral color combinations, $M=8$ $\mathrm{ms}, t(102)=1.47, p=.015, d=0.14$. This supports Wright, Boot, and Brockmole's (2015a) hypothesis that a slowdown for congruent color combinations (compared to incongruent or neutral combinations) is a prerequisite for observing the letter congruency effect.

\section{Discussion}

In the first experiment, we could show that task-irrelevant singleton items that have a feature in common with the target could delay the disengagement of attention. This means that in trials in which the colors of center circle and target circle are congruent, the disengagement was delayed compared to trials in which colors of center circle and target circle were nonmatching (delayed disengagement effect). These findings are in line with our hypotheses and replicate the pattern of results found by Wright, Boot, and Brockmole (2015a). Furthermore, for congruent color combinations we could confirm the letter congruency effect as observed by Wright, Boot, and

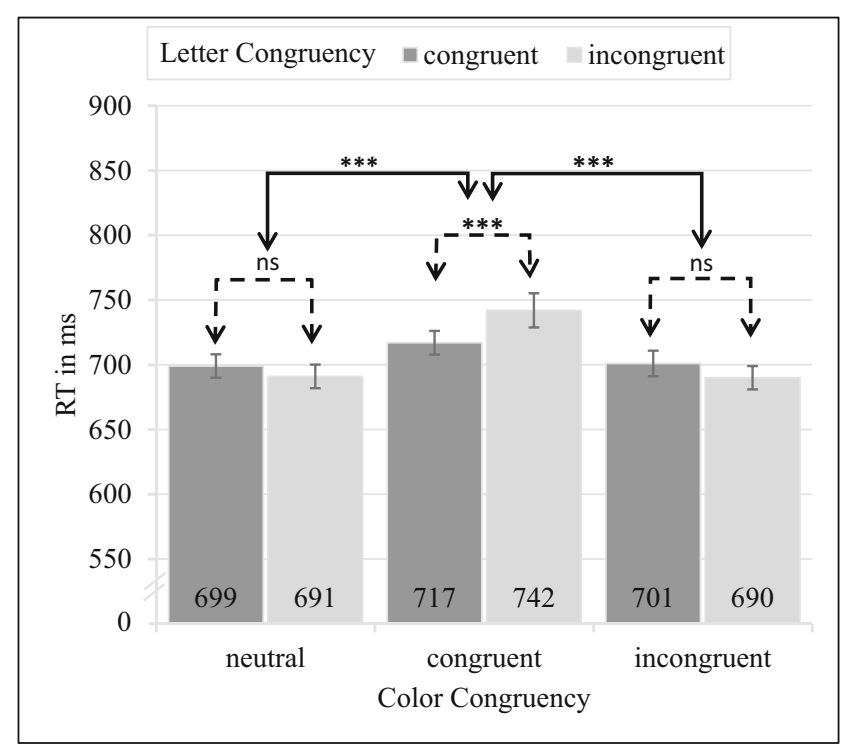

Fig. 2 Experiment 1, response times (RTs) for letter congruency as a function of color congruency. Comparisons regarding the letter congruency effect are indicated by the dashed lines. Error bars indicate betweensubject SEMs. $*=p<.05, * *=p<.01, * * *=p<.001$ 
Brockmole (2015a): responses are faster for matching center and target letters compared to incongruent center and target letters (letter congruency effect). They argue that this effect reveals a functional mechanism underlying the delayed disengagement consisting of a deeper level of visual processing of the center circle.

\section{Experiment 2 - Symbols}

In the first experiment, we replicated the pattern of results by Wright, Boot, and Brockmole (2015a). However, we were uncertain that a deeper level of processing was supposed to cause the delay in disengagement from the center circle. Using letters as crucial stimuli might not be ideal to show a general congruency effect as letters are especially familiar to observers and are recognized extremely quickly (Egeth \& Santee, 1981). Treisman and Gelade (1980) already stated that "letters have long been controversial units in perceptual theory." Letters are thus good candidates to be automatically processed as a whole requiring nearly no attention (Laberge, 1973). They further automatically recruit a sensory-motor brain network (James \& Gauthier, 2006) that might subserve in automatized responses. If the delayed disengagement effect indicates a general functional mechanism in the sense of deeper processing, a congruency effect should also be found by using unfamiliar symbols instead of very familiar letters. In other words, the letter congruency effect should generalize across familiarity. In order to avoid misunderstanding we stay with the expression letter congruency effect and add "(symbol)" to clarify that unfamiliar star-like shapes are used as symbols instead of letters.

Experiment 2 was supposed to replicate the same color congruency effect and letter congruency effect as in Experiment 1 and similar to Wright, Boot, and Brockmole (2015a). In Experiment 2 we opted for unfamiliar star-like shapes with six or eight edges that were hard to discriminate (as ensured in a pre-test comparison of triangles, squares, and star-like shapes). If delayed disengagement indicates deeper processing the letter (symbol) congruency effect is supposed to increase in magnitude, because the unfamiliar shapes should require deeper processing than familiar letters. If the letter (symbol) congruency effect remains unchanged, this is supportive of the color congruency effect being the result of deeper processing of the center circle. In summary, this experiment is thought to replicate both the color congruency effect and the letter (symbol) congruency effect from Experiment 1.

\section{Methods}

Participants Forty-two students (17 females and 25 males; 1927 years old, $M=21.7, S D=2.18$ ) from the Bundeswehr University Munich participated in this experiment. All participants had normal or corrected-to-normal vision. They provided informed consent and were given course credit as compensation for their participation.

We used exactly the same setting as in Experiment 1 except for two differences: the letters were replaced by symbols (each with $.6^{\circ}$ radius), and the center square was replaced by a fixation cross. Instead of deciding between ' $C$ ' or ' $\mathrm{O}$ ', participants had to decide whether a star shape had six or eight corners. All other circles contained different shapes (triangle, rotated triangle, square, rectangle, and diamond; cf. Fig. 3).

\section{Results}

We did not exclude any outliers based on RTs. Accuracy did interact with RT distributions, $F(2,82)=17.72, p<.001, \eta^{2}=$ .30 , but error rate was below $2 \%$ in all conditions. Further, we report median RTs per participant and condition.

To test the hypothesis that congruent colors lead to slower RTs compared to incongruent colors (color congruency effect), we first averaged over both congruent center target and incongruent center target color combination conditions. This means we used the same congruent, incongruent, and neutral color combinations as in Experiment $1 .^{2} \mathrm{We}$ applied a similar logic concerning the symbol combinations of the center and target circle: six-six corner combinations and eight-eight corner combinations are referred to as congruent combinations, six-eight or eight-six corner combinations are referred to as incongruent combinations.

To test for a slowdown in RTs for congruent symbol combinations as compared to incongruent symbol combinations, a repeated-measures ANOVA was calculated with color congruency (neutral, incongruent, vs. congruent) and letter (symbol) congruency (incongruent vs. congruent) as within-subject factors. The main effects for color congruency, $F(2,82)=49.4, p<.001, \eta^{2}=.55$, and letter (symbol) congruency, $F(2,41)=18.9, p<.001, \eta^{2}=.32$, were highly significant. The interaction was significant, $F(2,82)=28.5, p<.001, \eta^{2}=.41$. Similar to Experiment 1, we contrasted the color combinations and revealed significant differences for neutral $(659 \mathrm{~ms})$ versus congruent (687 ms) color combinations, $t(82)=4.26, p<$ $.001, d=0.38$ and incongruent $(661 \mathrm{~ms})$ versus congruent (687 ms) color combinations, $t(82)=3.80, p<.001, d=$ 0.35 , but not for neutral (659 ms) versus incongruent (661 $\mathrm{ms}$ ) color combinations, $t(82)=0.46, p=.65, d=0.029$ (see Fig. 4). These results support our hypothesis that only congruent color combinations delay responses.

\footnotetext{
${ }^{2}$ We did this because in the Wright, Boot, and Brockmole (2015a) study effect sizes were quite small and there were no significant differences between the before mentioned conditions, either in Wright, Boot, and Brockmole or in our raw RTs, $F(1,40)=3.42, p=.072, \eta^{2}=.079$.
} 

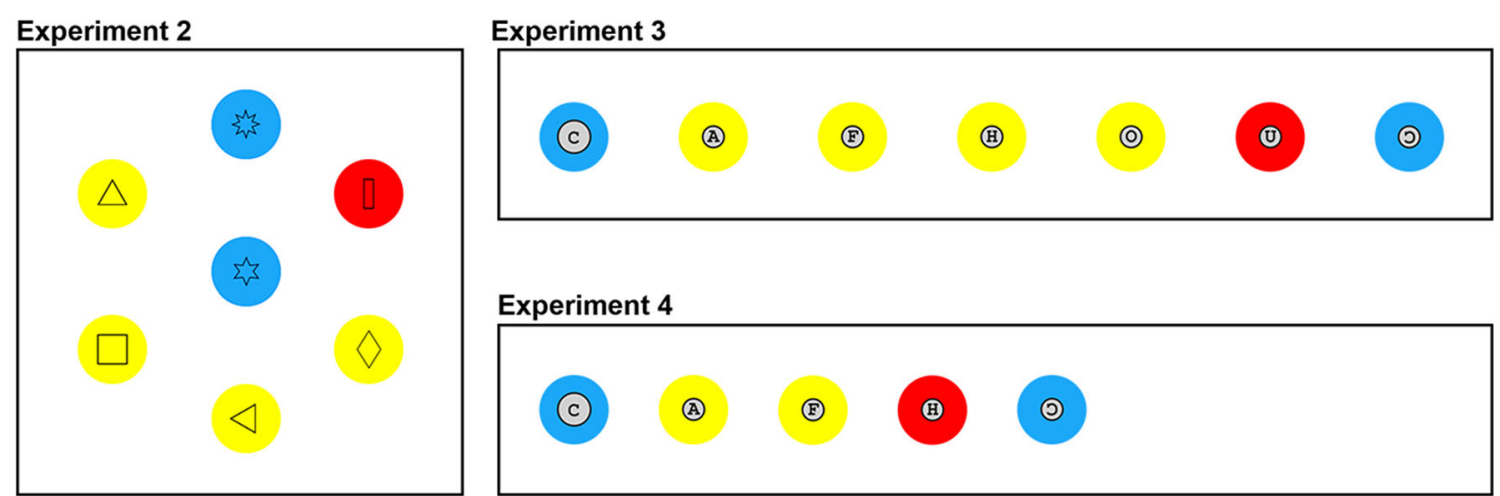

Experiment 5

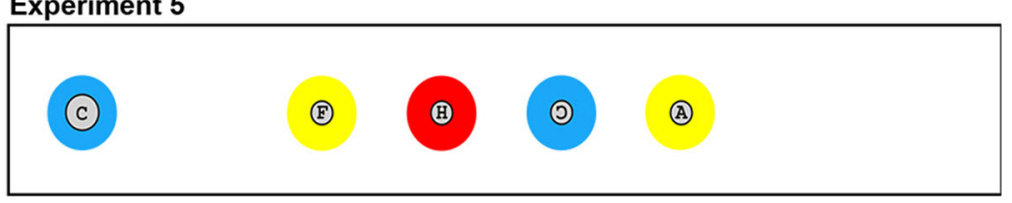

Fig. 3 Examples of the search display layout in Experiments 2, 3, and 4 and the "new" layout in Experiment 5. After the waiting period, the gray circles switched to different colors as displayed in Fig. 1. Participants were instructed to always search for the blue (or green) peripheral circle

Next, we analyzed the letter (symbol) congruency effect. There was a significant slowdown in RTs for incongruent versus congruent symbol congruency only for congruent color combinations, $M=35 \mathrm{~ms}, t(112)=5.92, p<$ $.001, d=0.44$ (see also Fig. 4), but not for incongruent color combinations, $M=1 \mathrm{~ms}, t(112)=1.10, p=.28, d=$ 0.014 , or neutral color combinations, $M=0 \mathrm{~ms}, t(112)=$ $0.27, p=.79, d=0.00$. This supports our findings from the previous experiment that a slowdown for congruent color combinations (compared to incongruent or neutral

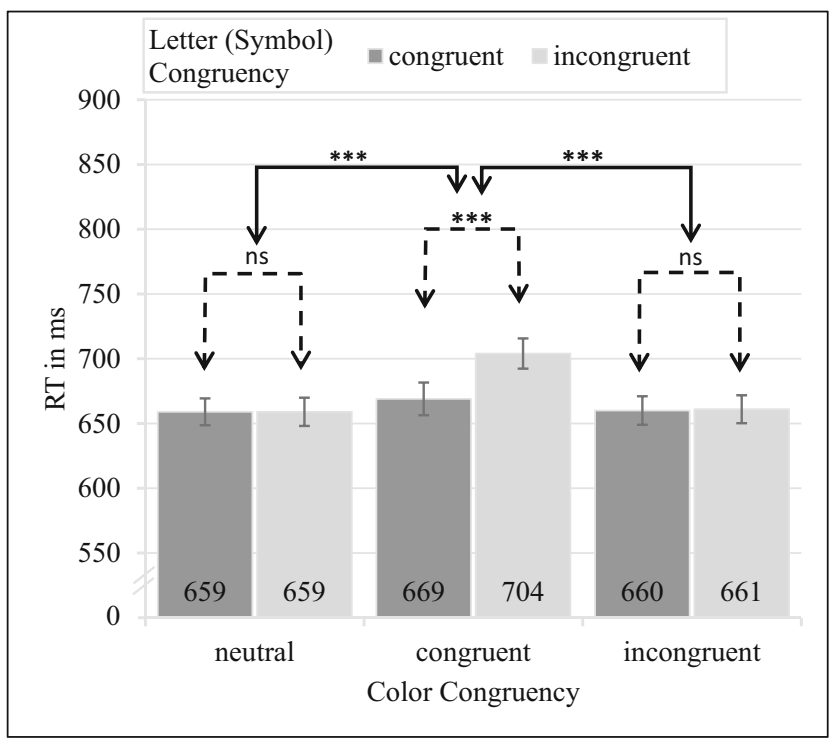

Fig. 4 Experiment 2, response times (RTs) for letter (symbol) congruency as a function of color congruency. Comparisons with regard to the letter (symbol) congruency effect are indicated by the dashed lines. Error bars indicate between-subject SEMs. $*=p<.05, * *=p<.01, * * *=p<.001$ and decide whether the target letter was a six- or eight-corner star-like shape (Exp. 2) or ' $\mathrm{C}$ ' or 'O' (Exp. 3 and 4). All other features of the other circles and the center circle were to be ignored

combinations) is a prerequisite for observing the letter (symbol) congruency effect.

\section{Discussion}

In Experiment 2, we could demonstrate that if center and target color match, response times are slowed (delayed disengagement) by about $28 \mathrm{~ms}$. This delay in disengagement is similar in size to Experiment 1 ( $35 \mathrm{~ms})$. This speaks in favor of a similar processing (of all relevant features) of the letters and the more complex unfamiliar symbols. Only if the colors match do non-matching center-target symbols slow RTs. The size of this letter (symbol) congruency effect of Experiment 2 ( $35 \mathrm{~ms}$ ) is also comparable to that of Experiment 1 ( $26 \mathrm{~ms}$ ). Overall, the results confirm the previous findings. Importantly, we could demonstrate that the functional delay in disengagement is not limited to letters but also extends to more complex unfamiliar stimuli.

\section{Experiment 3 - Seven linear circles}

In the previous experiments, we supported the hypothesis that disengagement of attention is automatically delayed in a search task when a fixated start item has features in common with the target item (Boot \& Brockmole, 2010). However, to our knowledge, this effect has only been demonstrated in a search paradigm with a circular search layout where the fixation circle is at the center and is surrounded by several peripheral circles (Biggs, Kreager, Gibson, Villano, \& Crowell, 2012; Blakely et al., 2012; Boot \& Brockmole, 2010; 
Brockmole \& Boot, 2009; Wang \& Theeuwes, 2018; Wright, Boot, \& Brockmole, 2015a; Wright, Boot, \& Jones, 2015b). In such layouts, the observer has to find the location of the target, which can be in any direction from the center circle. The goal of the third experiment was to generalize the findings to another search layout. Because the delayed disengagement effect might depend on factors that are still unknown, it is important to reduce noise in the search task. Circular displays introduce variance, as saccadic latencies (Heywood \& Churcher, 1980) and accuracy (Becker \& Jürgens, 1990) depend on the direction of the saccade. Crucially, the latencies are not influenced by their distance. Therefore, we chose a linear search layout that might also provide better generalizability to everyday tasks, as in everyday life we move our eyes most often horizontally. It is therefore possible that the delayed disengagement effect appears more robust (i.e., with less variance) in a search layout that only necessitates horizontal eye movements. Consequently, the layout was adapted in this experiment. All seven circles were now arranged in a straight line with the fixation circle always being the left-most one. This means that to perform the task, only left-to-rightsaccades have to be made. This also simplifies the search task in terms of spatial uncertainty: the direction of the to-beperformed saccade is known, only the distance remains uncertain.

\section{Methods}

Thirty-six students (19 females and 17 males; $19-28$ years old, $M$ $=23.14, S D=2.4$ ) from the Bundeswehr University Munich participated in this experiment. All participants had normal or corrected-to-normal vision. They provided informed consent and were given course credit as compensation for their participation.

In this experiment, we used the same stimulus display components and task setting as in Experiment 1, except that all circles were arranged on an imaginary straight line on the horizontal midline with equal distances of the circle centers to the upper and lower frame of the rectangular display (see Fig. 3). The distance between the circle centers was $5.6^{\circ}$. The starting circle was always the left-most circle. The letter in the center-circle was not masked again after the first saccade (see Fig. 3). Additionally, we now recorded eye movements with an EyeLink 1000 Plus (SR Research, Inc). Stimuli were presented on a ViewPixx 120-Hz Monitor at a distance of $68 \mathrm{~cm}$. We used saccadic latencies (SLs) to measure disengagement from the center circle and manual (button press) RTs (mRTs) to measure depth of processing of the center item. Similar to Wright, Boot, and Brockmole (2015a), saccadic latencies were calculated between the presentation of the search display and the start of the first saccade. Importantly, manual RTs were calculated differently to the previous experiments: beginning from the first fixation of the target to the button press. In the following, we report median times per participant and condition.

Trial exclusion An eye movement was classified as a saccade if its distance exceeded $0.2^{\circ}$ and velocity reached $30 \%$. The start of the first saccade had to be at the fixation circle (error rate was $5.3 \%$ ). We also excluded trials with backwards saccades $(9.2 \%)$, with a latency of the first saccade $<90 \mathrm{~ms}$ $(9.3 \%)$, trials that included a blink before the first saccade $(6.5 \%)$, and trials with an incorrect answer (2.7\%). We excluded all trials in which multiple saccades were necessary to reach an area of $3.8^{\circ}$ around the search target, ${ }^{3}$ leading to a loss of another $48.1 \%$ trials (This means $39.5 \%$ of all trials were left for analysis). The reasoning for this was that when an interim saccade lands on a non-target circle, the letter congruency effect could potentially not be observed just because the attentional set or the target representation in working memory was altered by this interim item "on the way" to the search target.

\section{Results}

Saccadic latency A repeated-measure ANOVA was calculated with color congruency (congruent, incongruent, or neutral) as a within-subject factor. The main effect for color congruency was highly significant, $F(2,72)=47.4, p<.001, \eta^{2}=.062$. The differences between the color combinations congruent versus incongruent and congruent versus neutral were found to be significant, $M=20 \mathrm{~ms}, t(72)=8.39, p<.001, d=0.56$, and, $M=20$ $\mathrm{ms}, t(72)=8.46, p<.001, d=.53$, and the difference between incongruent versus neutral, $M=0 \mathrm{~ms}, t(72)=0.07, p=.95, d=$ .00 , was not insignificant (see Fig. 5). These results support our hypothesis that congruent color combinations increase saccadic latency significantly.

Manual response times To test for a slowdown in mRTs for incongruent letter combinations as compared to congruent letter combinations, a repeated-measures ANOVA was calculated with color congruency (neutral, incongruent, or congruent) and letter congruency (incongruent or congruent) as within-subject factors. The main effect for color congruency was significant, $F(2,72)=$ $6.15, p=.003, \eta^{2}=.008$, and the main effect for letter congruency was not significant, $F(1,36)=1.00 p=.32, \eta^{2}=.001$. The interaction was also not significant, $F(2,72)=1.56, p=.22, \eta^{2}=$ .002. A slowdown for congruent color combinations (compared to incongruent or neutral combinations) is supposed to be a

\footnotetext{
3 This calculation was based on the reasonable concern of a reviewer that multiple saccade trials might potentially confound the effect as stated above. However, this approach (together with the re-calculation of mRTs) makes the results less comparable to Experiments 1 and 2 and other experiments without eye tracking. We therefore decided to report still report the more "classical" analyses (including multiple-saccade trials and with "classical" RT measures) in the Appendix. Notably, the pattern of results did not change (with one exception as noted below).
} 
prerequisite for the functional delayed disengagement in a sense of deeper processing of letters in both circles, and only then is the letter congruency effect expected as observed in Wright, Boot, and Brockmole (2015a).

To keep the results comparable, in post hoc t-tests we analyzed the letter congruency effect. There was no significant slowdown in mRTs for incongruent versus congruent letter combinations across color combination conditions (see Fig. 6) (congruent color combinations, $M=4 \mathrm{~ms}, t(95.6)=$ $.48, p=.63, d=.049$; incongruent color combinations, $M=-8$ ms, $t(95.6)=1.00, p=.32, d=.096$; neutral color combinations, $M=-13 \mathrm{~ms}, t(95.6)=1.61, p=.11, d=.16)$. We did not find a significant letter congruency effect in the linear design as expected from Experiments 1 and 2.

However, another difference of the linear compared to the circular design is that the second circle from the left is visually quite close to the starting circle and its presence might have influenced the deeper processing of the starting circle in some way (see Discussion). Such an influence would not possible if the second circle was the search target itself. Consequently, we performed the above ANOVAs and t-tests again only for the second circle from the left. We found similar main effects (color congruency: $F(2,70)=4.62, p=.013, \eta^{2}=.013$; letter congruency: $F(1,35)=0.076 p=.76, \eta^{2}=.00$; interaction: $\left.F(2,70)=0.48, p=.62, \eta^{2}=.001\right)$ with no significant differences in letter congruency for any color combination. ${ }^{4}$

\section{Discussion}

In Experiment 3, we used a linear instead of a circular arrangement of circles. Again, we found a delay in disengagement for matching colors in start and target circle. To the best of our knowledge, the present study is the first one to demonstrate the delayed disengagement effect in a linear arrangement of circles used as a search display. Former studies used circular arrangements of circles as search displays exclusively (Belopolsky et al., 2010; Biggs et al., 2012; Biggs \& Gibson, 2010; Blakely et al., 2012; Boot \& Brockmole, 2010; Born, Kerzel, \& Theeuwes, 2011a; Brockmole \& Boot, 2009; Wright, Boot, \& Brockmole, 2015a; Wright, Boot, \& Jones, 2015b). The delayed disengagement is exhibited by the saccadic latencies: Saccades are delayed, by $20 \mathrm{~ms}$, if and only if center and target color combinations match (congruent color combinations). Further, we assumed that only when the center circle color matches the target color, are $\mathrm{mRTs}$ prolonged, indicative of a deeper processing of the center circle. Contrary to our expectations, we did not find the letter congruency effect for matching center-target color combinations when using the linear search display. We

\footnotetext{
${ }^{4}$ In contrast to this, when including multiple-saccade trials and sticking to the classical calculation of RTs, we found a significant letter congruency effect for congruent color combinations. This is the only crucial difference between the two methods of analysis (see Appendix for details).
}

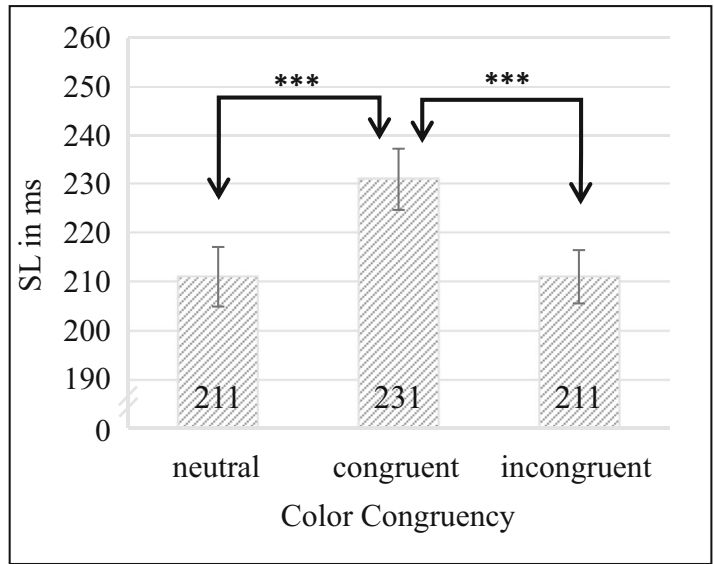

Fig. 5 Experiment 3, saccadic latency (SL) as a function of color congruency. Error bars indicate between-subject SEMs. $*=p<.05, * *=p<$ $.01, * * *=p<.001$

hypothesized that this could be due to the general inhibition of the starting circle in this design (which would imply that the delayed disengagement is not indicative of deeper processing in this configuration) or a blurring of the effect by the distractor circles adjacent to the target circle during processing. The letter congruency effect was also not exhibited when the analysis was limited to trials in which the circle right next to the starting circle was the search target. Since we found a normal delayed disengagement effect, it does not seem to be plausible that the fixation circle is inhibited in some way. It also does not seem to be the case that the circle adjacent to the fixation circle blurs the effect. One possibility might be that spatial uncertainty about the target item is determining

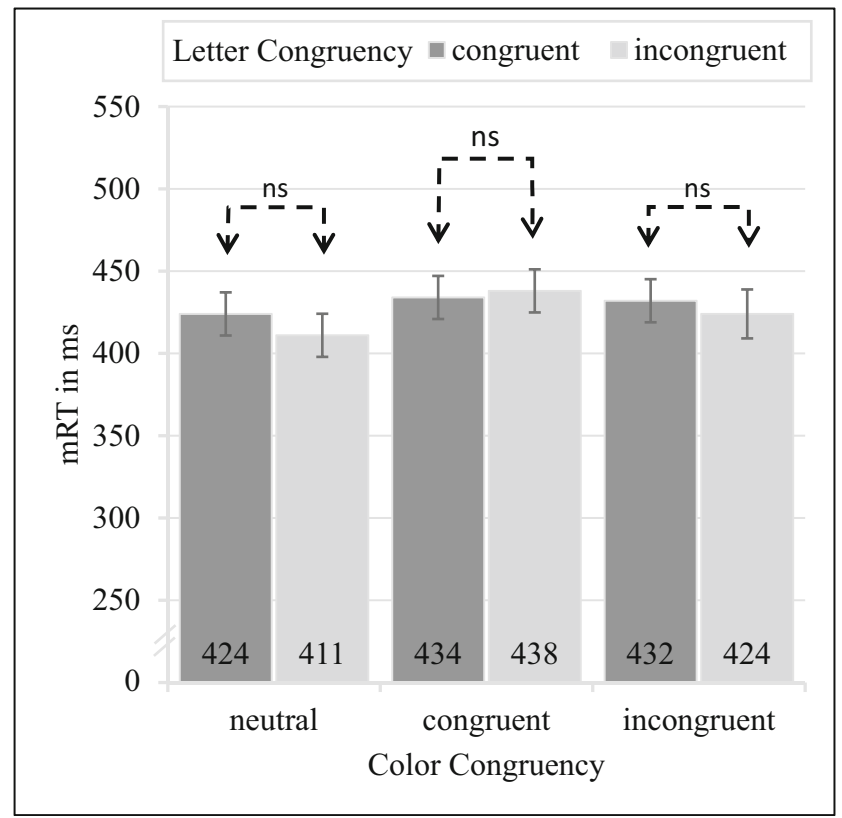

Fig. 6 Experiment 3, manual response times (mRTs) for letter congruency as a function of color congruency. Comparisons regarding the letter congruency effect are indicated by the dashed lines. Error bars indicate between-subject SEMs. $*=p<.05, * *=p<.01$, *** $=p<.001$ 
attentional dwell time on fixation items. A difference in our linear design compared to circular designs is that in the linear design it is always known in which direction the target is located (direction certainty), because it is always on the horizontal midline on the right of the fixation item. The only uncertainty revolves about its position along this line (distance uncertainty), whereas in circular designs, it is the reverse - the distance from fixation to target is always known (distance certainty), while the direction is uncertain (direction uncertainty). To test for this, we adapted the layout in the next experiment.

\section{Experiment 4 - Five linear circles}

In Experiment 4, we build on Experiment 3 to track the origin of the missing letter congruency effect - which is supposed to indicate deeper processing of the fixation item - in Experiment 3. Either it was too fragile and dissolved before the target was reached (as particularly long saccades were necessary), or the spatial direction uncertainty of the target is somehow a prerequisite for a deeper processing of the fixation item. Possibly, the search task is regarded as too easy and no deeper processing is necessary to quickly identify target objects along a straight line. To differentiate between these two possibilities, we changed the linear search layout in two ways. First, we reduced the number of circles from seven to five and ensured in a pretest that all possible search targets could be reached with the initial saccade. Additionally, we added the middle circle as a possible fixation circle. Half of the participants fixated the left-most circle, half of the participants fixated the middle circle. If the attenuation of deeper processing was caused by the direction certainty in the previous experiment, we should find a similar pattern of results to Experiments 1 and 2 with the middle fixation circle but not with the left-most fixation circle. If the attenuation of deeper processing was caused by the effect being fragile, we could potentially find the letter congruency effect with both the leftmost fixation and the middle fixation circle in the shortened linear arrangement.

To sum up, if we observe a letter congruency effect for both fixation positions, the number of the items (and length of saccades) was the origin of the null finding in Experiment 3. If we only observe a letter congruency effect for the middle fixation circle, the functionality of the delayed disengagement might be dependent on the search layout (i.e., direction uncertainty) and not generalizable across differing search layouts.

\section{Methods}

Forty-five students (14 females and 31 males; $19-34$ years old, $M=23.31, S D=3.53$ ) of the Bundeswehr University Munich participated in this experiment. All participants had normal or corrected-to-normal vision. They provided informed consent and were given course credit as compensation for their participation.

We used the same general setup as in Experiment 3, but instead of seven circles we only presented five circles. Additionally, the fixation circle was either the left-most circle or the middle circle (varied between subjects). For simplicity, instead of two target colors (green and blue) we only used one (blue). The hardware and software remained the same as in Experiment 3. As shown in Fig. 3 the five circles were positioned on a line with equal distances of the circle centers to the upper and lower frame of the rectangular display. We also decreased the distance between the circle centers from $5.6^{\circ}$ (Experiment 3) to $4.2^{\circ}$ to further shorten saccades (see Fig. 3).

Trial exclusion The start of the first saccade had to be at the fixation circle (error rate 10.5\%). We also excluded trials with backwards saccades (1.3\%), with a latency of the first saccade $<90 \mathrm{~ms}(5.3 \%)$, trials that included a blink before the first saccade (3.0\%), and trials with an incorrect answer (3.3\%). Similar to Experiment 3, we excluded all trials in which multiple saccades were necessary to reach an area of $2.1^{\circ}$ around the search target, leading to a loss of another $16.2 \%$ trials (this meant $67.6 \%$ of all trials left for analysis).

\section{Results}

Saccadic latency A repeated-measure ANOVA was calculated with fixation circle position (left or middle) as betweensubject factor and color congruency (congruent, incongruent, or neutral) as within-subject factors. The main effect for color congruency was highly significant, $F(2,86)=111.73, p<$ $.001, \eta^{2}=.24$. The main effect for fixation circle position, $F(1,43)=0.00, p=.97, \eta^{2}=.00$, was not significant. The interaction between color congruency and fixation circle position, $F(2,86)=2.75, p=.069, \eta^{2}=.006$, was not significant. As shown in Fig. 7, post hoc t-tests showed a delay in disengagement when the fixation circle had the same color as the target circle independent of position (color congruency conditions congruent vs. incongruent, $M=31 \mathrm{~ms}, t(86)=12.87, p<$ $.001, d=1.08$, congruent vs. neutral, $M=31 \mathrm{~ms}, t(86)=$ $13.03, p<.001, d=1.10$, and incongruent vs. neutral, $M=0$ $\mathrm{ms}, t(86)=0.16, p=.87, d=0.00)$.

Response times To test for a slowdown in mRTs for incongruent letter combinations as compared to congruent letter combinations, a repeated-measures ANOVA was calculated with fixation circle position (left or center) as between-subject factor and color congruency (neutral, incongruent, or congruent) and letter congruency (incongruent or congruent) as withinsubject factors. The main effects for color congruency, $F(2$, $86)=6.89, p=.002, \eta^{2}=.007$, and fixation circle position, $F(1,43)=4.75 p=.035, \eta^{2}=.083$, were significant. The main 


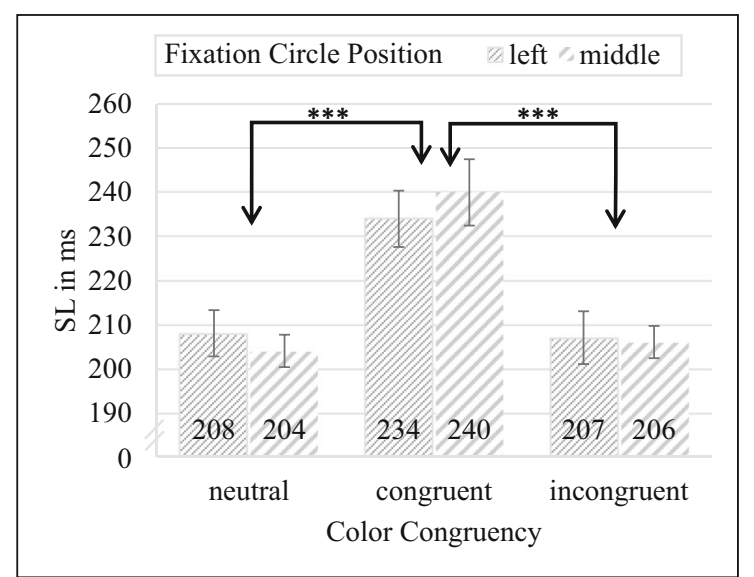

Fig. 7 Experiment 4, saccadic latency (SL) as a function of color congruency and fixation circle position. Error bars indicate between-subject SEMs. $*=p<.05, * *=p<.01, * * *=p<.001$

effect for letter congruency was not significant, $F(1,43)=$ $0.13, p=.72, \eta^{2}=.00$. The interaction between color congruency and letter congruency, $F(2,86)=8.99, p<.001, \eta^{2}=$ .011 , was significant. However, the interactions between color congruency and fixation circle position, $F(2,86)=2.29, p=$ $.11, \eta^{2}=.002$, letter congruency and fixation circle position, $F(1,43)=3.63, p=.063, \eta^{2}=.004$, and the three-way interaction between color congruency, letter congruency, and fixation circle position, $F(2,86)=0.52, p=.60, \eta^{2}=.001$, were not significant.

To test whether inner letters of the fixation circle were processed more deeply, in post hoc t-tests, we analyzed the letter congruency effect for both fixation circle positions individually. For the left fixation circle, there was no significant slowdown in mRTs for incongruent versus congruent letter combinations for congruent color combinations, $M=14 \mathrm{~ms}$, $t(118.8)=1.65, p=.10, d=0.26$, or neutral color combinations, $M=-13 \mathrm{~ms}, t(118.8)=1.56, p=.12, d=0.2$. We observed only a significant difference for incongruent color combinations, $M=19 \mathrm{~ms}, t(118.8)=2.34, p=.021, d=0.31$ (see also Fig. 8). In contrast, for the middle fixation circle position, there was a significant slowdown in mRTs for incongruent versus congruent letter combinations for congruent color combinations, $M=25 \mathrm{~ms}, t(118.8)=3.06, p=.003, d=$ 0.41 , but not for incongruent color combinations, $M=4 \mathrm{~ms}$, $t(118.8)=0.54, p=.59, d=0.082$, or neutral color combinations, $M=-2 \mathrm{~ms}, t(118.8)=.26, p=.79, d=0.036$ (see also Fig. 8). To sum up, there are differences between both fixation circle positions with only the middle fixation circle supporting the previously reported letter congruency effects.

In order to remain consistent with Experiment 3, we performed the above ANOVAs and t-tests again only for the adjacent circle of the left-most positioned fixation circle. We found that the letter congruency effect was significant (main effects: color congruency, $F(2,42)=0.16, p=.85, \eta^{2}=.001$, letter congruency, $F(1,21)=5.69 p=.027, \eta^{2}=.006$; interaction: $F(2,42)=1.74, p=.19, \eta^{2}=.005$.). In contrast to Experiment 3 , we found a significant letter congruency effect for congruent color combinations, $M=21 \mathrm{~ms}, t(62.2)=2.33, p$ $=.023, d=0.37$.

\section{Discussion}

In Experiment 4, we investigated whether the functionality of the delayed disengagement might be dependent on the fixation circle position and the number of linearly arranged circles. In between trials, participants fixated either the middle circle or the left-most circle and afterwards performed a saccade from this fixation circle to the target circle. For both starting positions, we found significant color congruency effects again: saccadic latencies were prolonged, when the colors of the fixation circle and the target circle matched (delayed disengagement). This was expected and is in line with the previous experiments. The magnitude of the effects remained comparable to Experiment 3 and to the findings of Wright, Boot, and Brockmole (2015a). Further, an observed letter congruency effect for both starting positions would have supported the hypotheses that, in Experiment 3, the number of circles (or length of saccades) was the origin of the null finding. An observed letter congruency effect only for the middle fixation circle, but not for the left fixation circle, would have supported the hypothesis that the claimed functionality of the delayed disengagement (Wright, Boot, \& Brockmole, 2015a) was dependent on the stimulus layout (i.e., direction uncertainty as a necessary prerequisite) and not generalizable to other layouts. On first sight, our overall findings seem to support the latter hypothesis, as for congruent fixation and target color combinations we only found a significant letter congruency effect for middle starting positions. The absence of a letter congruency effect for the left fixation circle seems to reproduce the results from Experiment 3.

We hypothesized that the letter congruency effect is fragile and was blurred during initial processing of the fixation circle, which was possibly caused (at least partially) by the adjacent circle. In the linear arrangements, there is a close proximity between the fixation circle and the next (possible target) circle (which is not the case in the "original" circular arrangements from Experiment 1 and Experiment 2). Due to their close proximity, the adjacent item might have also been processed on fixation - including its inner letter. If this is in fact true, it implies that there never was a "true" match of the attentional set (that encloses the fixation-circle-letter and at least part of the adjacent-circle-letter) and the target circle because the representation of the fixation-circle-letter was blurred by the letter of the adjacent circle. To test for this possibility, we only looked at trials in which the search target was right next to the fixation circle. In this configuration it should not have been possible that the attentional 


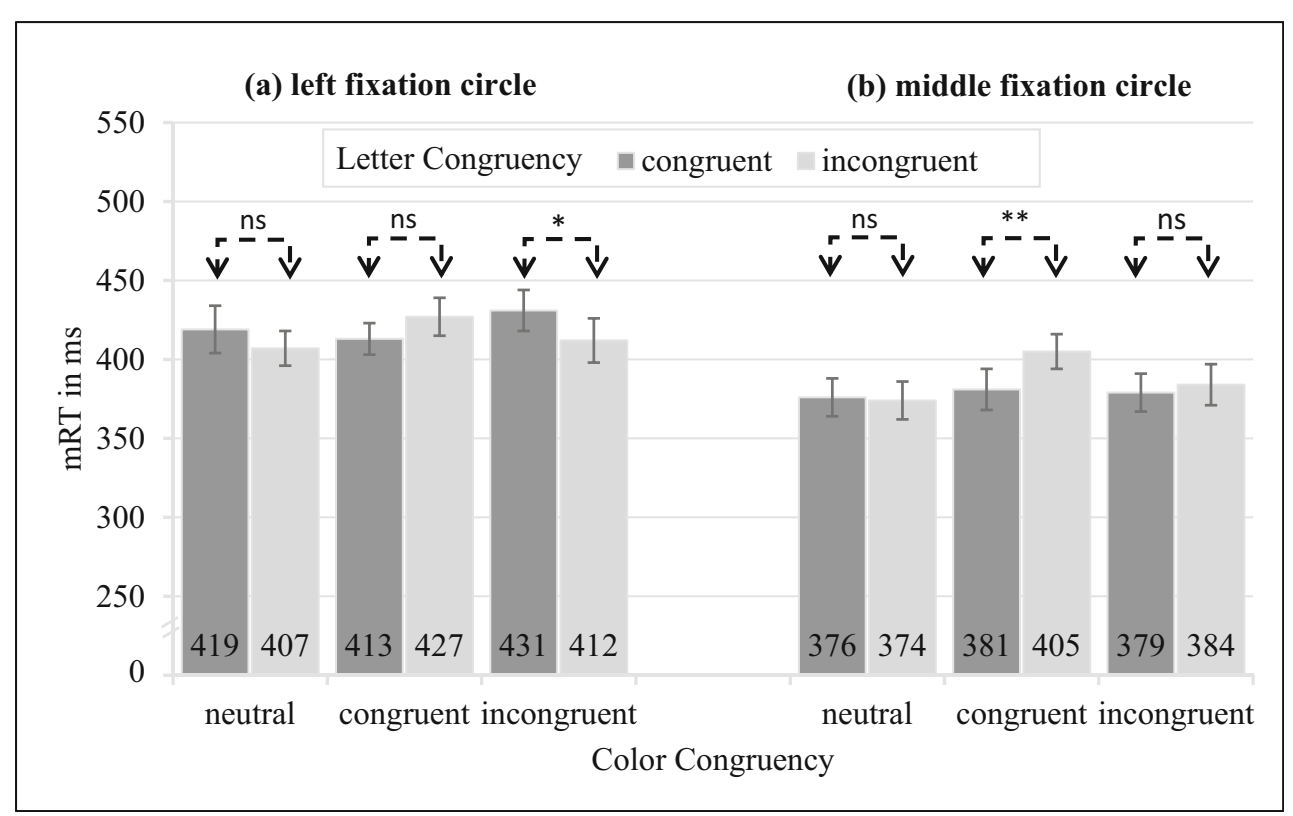

Fig. 8 Experiment 4, manual response times (mRTs) for left fixation position (a) and middle fixation position (b) for letter congruency depending on color congruency. Comparisons regarding the letter congruency effect are indicated by the dashed lines. Error bars indicate between-subject SEMs. $*=p<.05, * *=p<.01, * * *=p<.001$

shifted one position to the right (see Fig. 3). Thus, in this condition, there was a gap between the fixation and the first possible target. In addition, we have doubled the number of participants compared to the critical "left-most fixation circle" condition of Experiment 4. Given these changes, we can now investigate the hypotheses by analyzing occurrences of letter congruency (over all trials and) especially at the second target circle position (see circles with inner letter ' $F$ ' in Fig. 3, Experiments 4 and 5). If the representation of the fixation is in fact blurred by the adjacent circle, there should be an observable letter congruency effect only in the 'gap' condition because there is no adjacent circle to blur the effect. If the former null result is not due to blurring of the fixation circle but to more unspecific reliability issues, like statistical power, we expect similar findings in both conditions (letter congruency effect present or absent alike). Additionally, as the critical layout differences were presented on a within-subject basis in this experiment, we expect to get more consistent findings.

\section{Methods}

Forty-four students (ten females and 34 males; $19-41$ years old, $M=25.36, S D=4.25$ ) of the Bundeswehr University Munich participated in this experiment. All participants had normal or corrected-to-normal vision. They provided informed consent and were given course credit as compensation for their participation.

We used the same general setup as in Experiment 4 with the left-most circle as fixation position. In half of the blocks, we introduced a new layout in which all search circles were directly adjacent to the fixation circle but all target circles were 
shifted one position to the right, so there was now a gap $\left(8.4^{\circ}\right)$ between the fixation circle and the first search circle ('gap' condition, see Fig. 3). The other half of the blocks were an exact replication of Experiment 4 in the left-most fixation position ("no gap" condition). In addition, participants now completed 480 instead of 360 trails in four instead of three blocks. The order of the blocks was always alternating, and the starting block was counterbalanced between the participants.

Trial exclusion The start of the first saccade had to be at the fixation circle (error rate is $9.9 \%$ ). We also excluded trials with backwards saccades (4.2\%), with a latency of the first saccade $<90 \mathrm{~ms}(5.9 \%)$, trials that included a blink before the first saccade $(2.5 \%)$ and trials with an incorrect answer (5.0\%). Similar to Experiment 3 and 4, we excluded all trials in which multiple saccades were necessary to reach an area of $2.1^{\circ}$ around the search target, leading to a loss of another $26.0 \%$ trials (this means $65.6 \%$ of all trials were left for analysis).

\section{Results}

Saccadic latency A repeated-measure ANOVA was calculated with the factors gap condition (no gap or gap) and color congruency (congruent, incongruent, or neutral) as within-subject factors. The main effect for color congruency was highly significant, $F(2,84)=106.87, p<.001, \eta^{2}=.13$. The main effect for gap condition, $F(1,42)=6.82, p=.012, \eta^{2}=.005$, and the interaction between color congruency and first search circle position, $F(2,84)=3.11, p=.050, \eta^{2}=.001$, were also significant. As shown in Fig. 9, post hoc t-tests showed a delay in disengagement when the fixation circle had the same color as the target circle independent of gap condition (color congruency conditions congruent vs. incongruent, $M=30 \mathrm{~ms}, t(84)=$ $12.93, p<.001, d=0.79$, congruent vs. neutral, $M=29 \mathrm{~ms}$, $t(84)=12.38, p<.001, d=0.74$, and incongruent vs. neutral, $M=1 \mathrm{~ms}, t(84)=0.54, p=.59, d=0.031$.)

Response times To test for a slowdown in mRTs for incongruent letter combinations as compared to congruent letter combinations, a repeated-measures ANOVA was calculated with the factors gap condition (gap or no gap), color congruency (neutral, incongruent, or congruent) and letter congruency (incongruent or congruent) as within-subject factors. The main effect for gap condition, $F(1,42)=30.09 p<.001, \eta^{2}=.022$, was significant. The main effects for letter congruency, $F(1$, $42)=0.012, p=.92, \eta^{2}=.00$, and color congruency, $F(2,84)$ $=0.98, p=.38, \eta^{2}=.00$, were not significant. The interaction between color congruency and letter congruency, $F(2,84)=$ $30.85, p<.001, \eta^{2}=.014$, was significant. However, the interactions between color congruency and gap condition, $F(2,84)=0.86, p=.43, \eta^{2}=.00$, letter congruency and gap condition, $F(1,42)=1.52, p=.23, \eta^{2}=.00$, and the three-way

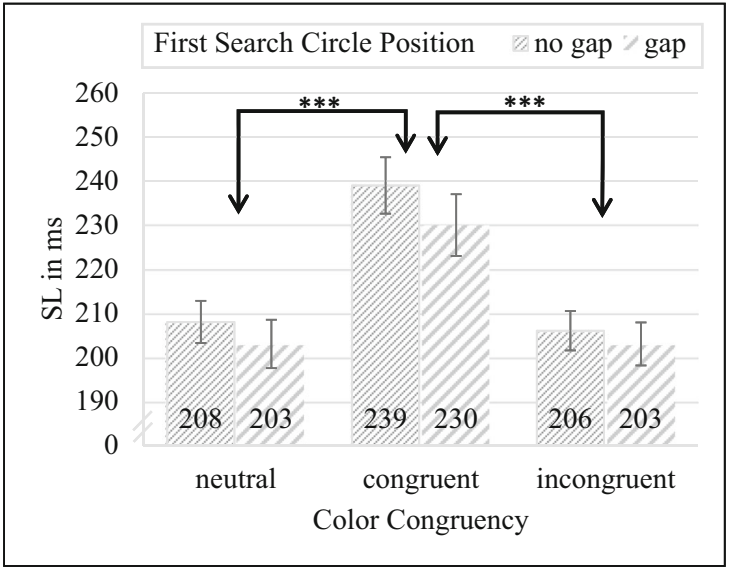

Fig. 9 Experiment 5, saccadic latency (SL) as a function of color congruency and fixation circle position. Error bars indicate between-subject SEMs. $*=p<.05, * *=p<.01, * * *=p<.001$

interaction between color congruency, letter congruency, and gap condition, $F(2,84)=0.99, p=.38, \eta^{2}=.00$, were not significant.

To test whether inner letters of the fixation circle were processed more deeply, in post hoc t-tests we analyzed the letter congruency effect for both gap conditions individually. For the "no gap" condition, there was a significant slowdown in mRTs for incongruent versus congruent letter combinations for congruent color combinations, $M=$ $28 \mathrm{~ms}, t(145)=4.28, p<.001, d=0.38$, but not for neutral color combinations, $M=4 \mathrm{~ms}, t(145)=0.55, p$ $=.58, d=0.059$ (see also Fig. 10). Furthermore, we observed a significant difference for incongruent color combinations, $M=-18 \mathrm{~ms}, t(145)=2.68, p=.008, d=0.25$. For the "gap" condition, there was a significant slowdown in mRTs for incongruent versus congruent letter combinations for congruent color combinations, $M=18 \mathrm{~ms}$, $t(145)=2.68, p=.008, d=0.24$, but not for neutral color combinations, $M=-12 \mathrm{~ms}, t(145)=1.83, p=.067, d=$ 0.18 . Furthermore, we again observed a significant difference for incongruent color combinations, $M=-15 \mathrm{~ms}$, $t(145)=2.35, p=.020, d=0.23$. To sum up, there are no significant differences between both first search circle positions, both supporting the previously reported letter congruency effects. ${ }^{5}$

\footnotetext{
${ }_{5}^{5}$ As an additional analysis, we calculated an overall ANOVA for the letter congruency effect with the subjects from Experiments 4 (left-most fixation circle) and 5 ("no gap" condition), as these layouts were identical. The main effects for letter congruency, $F(1,64)=0.021, p=.87, \eta^{2}=.00$, and color congruency, $F(2,128)=0.090, p=.91, \eta^{2}=.00$, were not significant. The interaction between color congruency and letter congruency, $F(2,128)=$ $24.07, p<.001, \eta^{2}=.017$, was significant. In post hoc tests, the letter congruency effect was significant for incongruent versus congruent letter combinations for congruent color combinations, $M=24 \mathrm{~ms}, t(157)=4.36, p<.001, d=$ 0.34 , but not for neutral color combinations, $M=-8 \mathrm{~ms}, t(157)=1.27, p=.21, d$ $=0.11$. Furthermore, we observed a significant difference for incongruent color combinations, $M=-18 \mathrm{~ms}, t(157)=3.41, p<.00, d=0.26$.
} 


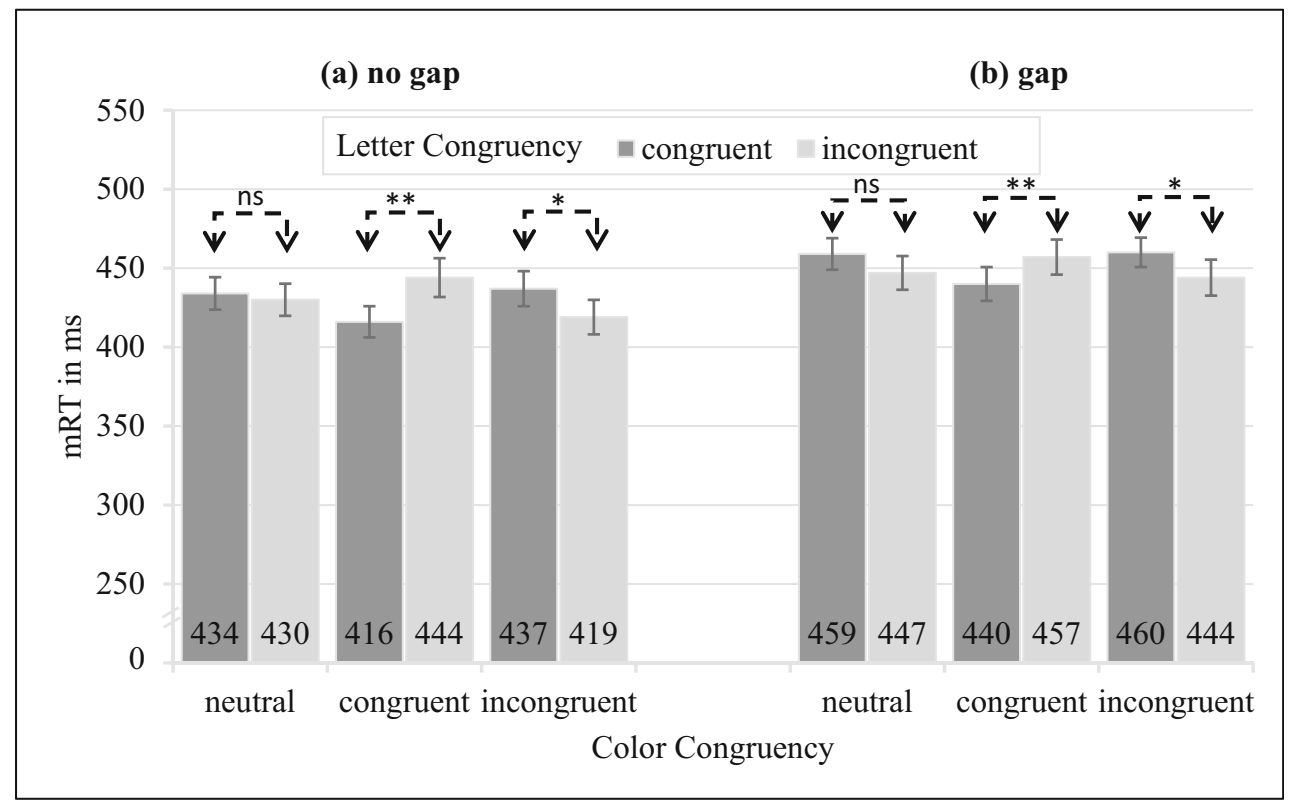

Fig. 10 Experiment 5, manual response times (mRTs) for first search circle position with no gap (a) and with gap (b) for letter congruency depending on color congruency. Comparisons regarding the letter

We performed the above ANOVAs and t-tests again only for the first target circle from the left in the gap condition and the second target circle from the left in the no gap condition. This meant that we had the same distance from fixation to the target circle in both conditions. We found the main effect for color congruency, $F(2$, $84)=8.40, p<.001, \eta^{2}=.006$, and interactions of color congruency and letter congruency: $F(2,84)=12.43, p<$ $.001, \eta^{2}=.013$, all other effects were not significant). The letter congruency effect for congruent color combination was significant for the "no gap" condition, $M=29 \mathrm{~ms}$, $t(219)=2.72, p=.008, d=0.35$, but not for the "gap" condition, $M=19 \mathrm{~ms}, t(219)=1.84, p=.067, d=0.22)$.

\section{Discussion}

In Experiment 5, we investigated whether the missing letter congruency effect from Experiments 3 and 4 was due to a blurring of the representation of the fixation item. We adapted the design so that there was a circle-sized gap between the fixation circle and the first possible target circle in half of the trials. For both gap conditions, we found significant color congruency effects: saccadic latencies were prolonged when the colors of the fixation circle and the target circle matched (delayed disengagement). This was expected and is in line with the previous experiments. The magnitude of the effects remained comparable to Experiments 3 and 4. Additionally, the results reveal significant letter congruency effects in both gap conditions (gap and no gap) for congruent color congruency conditions. These results support the postulation from congruency effect are indicated by the dashed lines. Error bars indicate between-subject SEMs. $*=p<.05, * *=p<.01, * * *=p<.001$

Wright, Boot, and Brockmole (2015a) that the delay in disengagement (when the fixation item color matches the target item color) is due to a deeper processing of the fixation item. The findings thereby provide evidence that this hypothesized underpinning of the delayed disengagement effect holds generally also true in linear display layouts and is not, in principle, dependent on the exact display configuration.

Contrary to our expectations, when limiting the analysis to the second target circle, there was a significant letter congruency effect only for the no gap condition; that is, when there is a gap between the fixation item and target item (instead of another possible target item), the letter congruency effect could not be clearly resolved. But since the interaction of gap position and letter congruency was not significant, we do not assume these are systematical differences in gap position. Nevertheless, this result might further indicate that our initial explanation of the null findings in Experiments 3 and 4 (i.e., caused by a blurring of the representation of the fixation item) was not applicable.

Of note, there also seems to be a surprisingly consistent "reverse" letter congruency effect for incongruent color combinations. This means that, whenever fixation item color and target item color do not match (i.e., are incongruent) and there is no delay in disengaging from the fixation item, a non-matching fixation-letter and target-letter combination delays manual responses. As this pattern is similar in Experiments 3 and 4 (albeit only significant in Experiment 4), further discussion can be found in the General discussion below. 


\section{General discussion}

The goal of the present study was to test the generalizability of the delayed disengagement effect: responses to search targets are slower when the irrelevant fixation object has features in common with the target object (Belopolsky et al., 2010; Biggs et al., 2012; Biggs \& Gibson, 2010; Blakely et al., 2012; Boot \& Brockmole, 2010; Brockmole \& Boot, 2009; Folk et al., 1992; Theeuwes, 1994; Wright, Boot, \& Brockmole, 2015a; Wright, Boot, \& Jones, 2015b). In particular, we tested the generalizability of its proposed functional nature in terms of deeper processing of the fixation item (Wright, Boot, \& Brockmole, 2015a; Wright, Boot, \& Jones, 2015b), with unfamiliar symbols rather than letters and in a linear search layout in addition to the predominantly employed circular search layout. The hypothesis that the delayed disengagement has a functional reason was originally proposed by Wright, Boot, and Brockmole (2015a). These authors showed that when participants had to respond to a letter in the target item, responses are delayed if the letter in the task-irrelevant fixation item does not match the letter of the target object (manual responses were faster when fixation-circle-letter and targetcircle-letter matched). They concluded that the letter in the fixation circle must have been processed and thus, the disengagement from the fixation circle was delayed because it was processed more deeply (i.e., the inner letter is identified). This was the case only when fixation and target circle were the same color.

In the present study, we consistently found this general delayed disengagement effect (delay in responses when fixation and target colors matched) across all five experiments. This finding helps to generalize delayed disengagement effects, as they do not seem to be dependent on the layout of the search display. Notably, the influence of the effect seems to be quite stable across search layouts as it was rather similar in magnitude in all five experiments ( $20 \mathrm{~ms}$ to $35 \mathrm{~ms}$ ), serving as further quantification of its effect size.

Further, in Experiment 1, we successfully replicated the letter congruency effect (which is supposed to indicate a functional nature of the delayed disengagement) with the same letters used by Wright, Boot, and Brockmole (2015a). In Experiment 2, instead of simple letters, participants had to distinguish between two presumably unfamiliar star-like shapes that were hard to discriminate. We found comparable results to the first experiment, supporting the delayed disengagement effect in general and found further evidence supporting its hypothesized functional nature in particular. Thus, we corroborated the previous findings and were, as far as we know, the first to show the letter congruency effect (hypothesized to indicate the functional reason for the delayed disengagement effect) with unfamiliar symbols.

In the linear design from Experiments 3 and 4 (left-most fixation position) we did not observe a letter congruency effect (however, it was evident in Experiment 5). We originally hypothesized that the letter congruency effect was fragile and was blurred during initial processing of the fixation circle, which might have been caused at least partially by the adjacent circle. Due to the close proximity of the next circle to the fixation circle (as compared to the circular arrangements in Experiments 1 and 2), the adjacent object might have also been processed unintentionally - including its inner letter. If this were the case it would imply that there was never a true "match" of the attentional set (that encloses the fixation-circleletter and at least part of the adjacent-circle-letter) and the target circle because the representation of the fixation-circleletter got blurred by the letter of the adjacent circle. To further investigate this possibility, we conducted the fifth experiment, in which there was a circle-wide gap between the fixation circle and the first possible target circle. In this new experiment, we found a consistent letter congruency effect for both gap conditions, indicating that blurring of the fixation item was likely not the cause of the absent letter congruency effect in Experiments 3 and 4.

We found consistent letter congruency effects in Experiment 5 and in a joint reanalysis of Experiments 4 and 5. These ambiguous results give rise to the possibility that the letter congruency effect tends to become more unreliable if the layout necessitates longer saccades, that is, it might have been that the blurring and attenuation of the attentional set covaries with the length of the saccades (i.e., over time). This would imply that the effect of deeper processing (as presumed to be indicated by delayed disengagement) would be exhibited most consistently when short saccades are to be performed (and for longer saccades, it would be harder to show significantly). Note that we do not propose that the delayed disengagement effect was influenced by the longer saccades, as saccadic latencies are typically not affected by the length of the saccades (Heywood \& Churcher, 1980), but one might argue that the specific letter congruency effect (that is apparent in manual response times) was affected. There are some indications that speak in favor of this speculation. Even if only one-saccade trials are included, the average saccade distances were longer in Experiment $3\left(16.8^{\circ}\right)$, in Experiment $4\left(8.4^{\circ}\right.$; left-most fixation circle condition), and in Experiment 5 (10.5 ; gap condition) compared to the saccade distance Wright, Boot, and Brockmole (2015a) reported $\left(7,8^{\circ}\right)$ in connection with their circular search display with a center fixation circle. A further indication is that we replicated the letter congruency effect in a condition based on a comparable saccade distance $\left(6.3^{\circ}\right)$ (Experiment 4; middle fixation circle condition) even with a lower sample size. However, especially horizontal saccades are performed extremely fast (Becker \& Jürgens, 1990) and the difference between longer and shorter saccades is in the order of some milliseconds. Thus, it is implausible to assume that the length of saccades can fully account for the reliability of the letter congruency effects. However, based solely on our 
joint results, it seems that the letter congruency effect is the most stable, the shorter the to-be-performed-saccades are. Notably, there seems to be a consistent "reverse" letter congruency effect for incongruent color combinations: Whenever fixation item color and target item color do not match, that is, there is no delay in disengaging from the fixation item, a nonmatching fixation-letter and target-letter combination delays manual responses. This general pattern can be generally observed across all linear designs with a left fixation position and the effect sizes seem to be comparable (Exp. 3: $8 \mathrm{~ms}$, Exp. 4: $19 \mathrm{~ms}$, Exp. 5: $17 \mathrm{~ms}$ ), although the difference was only significant in Experiments 4 and 5.

In their original experiment, Wright and colleagues (2015a) offered (but quickly discarded) an alternative explanation for the delay in disengagement (for congruent fixation-target colors): They speculated that center items might be inhibited and only after successful inhibition of the fixation item (which takes additional time), the saccade is executed. It could be that - possibly only in linear layouts - the fixation item is always inhibited to some extent. A reason for inhibition in this case could be that a linear layout with a left starting circle is spatially more ordered. The visual system can easily tune itself to inhibit/suppress certain display locations or even whole regions where a distracting object is likely to appear (cf. Gaspelin, Leonard, \& Luck, 2015; Sauter, Liesefeld, Zehetleitner, \& Müller, 2018; Wang \& Theeuwes, 2018) but probably only when they are spatially clearly separable and in the periphery. It could be that in the present study's Experiments 4 and 5, the fixation item was inhibited - including its center letter - when its prominent feature was clearly spatially separable and not task relevant (i.e., in the incongruent condition). However, since this inhibition had to be extremely fast to not delay the disengagement from the fixation item (compared to the neutral condition), this is to be taken as a first speculation and should be matter of other investigations.

\section{Conclusion}

Delayed disengagement could be demonstrated in several experiments and with different types of stimuli (letters or symbols) and two different types of layout arrangements (linear and circular layout). Delayed disengagement is found to be a stable effect across all experiments. To the best of our knowledge, the present study is the first to consistently demonstrate the delayed disengagement effect across multiple search layouts and with unfamiliar target symbols. Similarly, the proposed generality of the functional explanation of the delayed disengagement (in terms of deeper processing of the fixation items) seems to be accurate. This interpretation finds support by utilizing a search space with a circular as well as linear layout. For experiments or conditions in which we did not reveal an effect, it may be that the attentional set that is active during the delay before disengaging the fixation is fragile, short lived and for this reason functional effects are weak (especially for layouts necessitating longer saccades). We offered several explanations of the absence of the letter congruency effect in Experiments 3 and 4 that seemed superficially plausible to us beyond concerns of statistical power: the inhibition of the processing of the features of the fixation circle, that fixation circle and adjacent circle share attentional focus ("blurring"), but none of them was able to fully account for the given effect pattern. Based on the stable letter congruency effects from Experiment 5 and a joint reanalysis, our results support the view that delayed disengagement can be interpreted in terms of deeper processing of the fixation item. Therefore, the proposed functionality of delayed disengagement seems to be generalizable to non-circular display layouts but might be more reliable in classical circular arrangements.

Acknowledgements We would like to thank Tobias Bauer, Yuping Jiang, Miriam Klink, and Linus Rüber for their help with the data collection.

\section{Appendix}

In the analyses above, we removed all trials in which multiple saccades were executed to reach the search target because of a potential contamination of the attentional set at the landing of interim saccades. In addition, we calculated manual response times from the time the saccade reached the search target and the following button press. While these two measures are reasonable in an eyetracking study, the effects are not easily compared to noneye-tracking studies, as saccade onset and landing are not measurable. We therefore offer the alternative "classical" calculations here. This means, in the calculations below, we included all trials, regardless of how many saccades were made and we calculated the response times classically from the onset of the search display to the button press.

\section{Classical analyses and results for Experiment 3 data}

Trial exclusion We included all eye movements where the distance exceeded $0.2^{\circ}$ and its velocity reached $30 \%$. Furthermore, the start of the first saccade had to be at the fixation circle (error rate is $5.6 \%$ of total trials). We also excluded trials with backwards saccades ( $9.2 \%$ of total trials), with a latency of the first saccade $<90 \mathrm{~ms}$ ( $9.3 \%$ of total trials) and trials that included a blink before the first saccade $(6.9 \%$ of total trials). In sum, $25.8 \%$ of all trials were excluded from analyses. In some trials, one saccade was not enough to reach the search target and we included all correct trials independent of how many saccades were necessary to reach the target.

Saccadic latency To test our hypothesis that saccadic latency (SL) was slower for congruent color combinations, a repeated- 
measure ANOVA was calculated with color congruency (congruent, incongruent, or neutral) and letter congruency (incongruent or neutral) as within-subject factors. The main effect for color congruency is highly significant, $F(2,72)=50.67, p$ $<.001, \eta^{2}=.59$. The main effect for letter congruency is not significant, $F(1,36)=0.17, p=.69, \eta^{2}=.005$. The interaction is not significant, $F(2,72)=0.87, p=.42, \eta^{2}=.024$. The contrasts of the color combinations congruent versus incongruent and congruent versus neutral were found to be significant, (mean difference $19 \mathrm{~ms}) t(72)=7.5, p<.001, d=0.47$, and (mean difference $25 \mathrm{~ms}) t(72)=9.56, p<.001, d=0.64$, and the contrast incongruent versus neutral, (mean difference $5 \mathrm{~ms}) t(72)=2.06, p=.043, d=0.15$, was insignificant. These results support our hypothesis that congruent color combinations delay saccadic latency significantly.

Response times In the following, we report median correct RTs per participant and condition. RTs were calculated from the onset of the search display to the button press. To test a slowdown in RTs for congruent letter combinations as compared to incongruent letter combinations, a repeated-measures ANOVA was calculated with color congruency (neutral, incongruent, or congruent) and letter congruency (incongruent or congruent) as within-subject factors. The main effects for color congruency, $F(2,72)=11.63, p<.001, \eta^{2}=.24$, and letter congruency, $F(2,36)=4.68 p=.037, \eta^{2}=.12$, are significant. The interaction is not significant, $F(2,72)=$ $1.28, p=.29, \eta^{2}=.034$. In post hoc t-tests, we analyzed the letter congruency effect. Contrary to expectations, there was no significant slowdown in RTs for incongruent versus congruent letter combinations across color combination conditions (incongruent color combinations, $t(107)=1.68, p=$ $.096, d=0.13$ congruent color combinations, $t(107)=0.20$, $p=.84, d=0.01$, neutral color combinations, $t(107)=1.96, p$ $=.053, d=0.16$. We did not find a significant letter congruency effect in the linear design as we found in Experiments 1 and 2. The ANOVA's main effect and post hoc tests even seem to point in the opposite direction.

However, another difference of the linear compared to the circular design is that the second circle from the left is visually quite close to the starting circle and its presence might have influenced the deeper processing of the starting circle in some way. Such an influence is not possible if the second circle is the search target itself. Consequently, we performed the above ANOVAs and t-tests again only for the second circle from the left. We found that the color congruency effect is still prevalent (main effects: color congruency, $F(2,72)=9.7, p<.001$, $\eta^{2}=.21$, letter congruency, $F(1,36)=.45 p=.51, \eta^{2}=.012$; interaction: $\left.F(2,72)=2.56, p=.084, \eta^{2}=.066\right)$ with significant differences only for neutral (648 ms) versus congruent (684 ms) color combinations, $t(72)=4.15, p<.001, d=0.33$, and incongruent $(655 \mathrm{~ms})$ versus congruent $(684 \mathrm{~ms})$ color combinations, $t(72)=3.36, p=.001, d=0.28$. Additionally, we found a significant letter congruency effect for congruent color combinations, $t(106)=2.04, p=.044, d=0.23$. Further, nonsignificant results are not reported. This finding is supportive for a functional process as the basis for the delay in disengagement from the fixation circle but seems to be restricted to a target circle that is within the attentional focus of the fixation circle. If the target circle is outside the attentional focus, the supposed deeper processing seems to lose functional value perhaps due to distortions.

\section{Classical analyses and results for Experiment 4 data}

Trial exclusion First, we included all correct trials no matter how many saccades were made. We included saccades where the distance exceeded $0.2^{\circ}$ and its velocity reached $30 \%$. Furthermore, the start of the first saccade had to be at the fixation circle (error rate is $10.47 \%$ of total trials). Trials with first saccades in wrong direction (3.6\% of total trials), latency of the first saccade $<90 \mathrm{~ms}$ ( $5.34 \%$ of total trials), and blink before first saccade ( $3.21 \%$ of trials) were also not included. In sum, $21.66 \%$ of total trials were excluded from analyses. Further, we report medians RTs per participant and condition.

Saccadic latency To test our hypothesis, that saccadic latency (SLs) was slower for congruent color combinations, a repeated-measure ANOVA was calculated with fixation circle position (left or middle) as between-subject factor and color congruency (congruent, incongruent, or neutral) and letter congruency (incongruent or neutral) as within-subject factors. The main effect for color congruency is highly significant, $F(2,86)=120.68, p<.001, \eta^{2}=.73$. The main effect for letter congruency, $F(1,43)=0.23, p=.64, \eta^{2}=.005$, and fixation circle position, $F(1,43)=0.012, p=.91, \eta^{2}=.00$, is not significant. The interaction between letter congruency and fixation circle position, $F(1,43)=5.75, p=.021, \eta^{2}=.12$, is significant. However, the interactions between color congruency and fixation circle position, $F(2,86)=2.82, p=.065$, $\eta^{2}=.017$, color congruency and letter congruency, $F(2,86)=$ $0.025, p=.98, \eta^{2}=.001$, and color congruency, letter congruency, and fixation circle position, $F(2,86)=0.025, p=.98, \eta^{2}$ $=.001$, are not significant. Our results indicate slowing in disengagement when the fixation circle had the same color as the target circle independent of position. We contrasted the letter congruency conditions congruent versus incongruent (mean difference $30 \mathrm{~ms}) t(86)=13.43, p<.001, d=1.061$, congruent versus neutral (mean difference $30 \mathrm{~ms}) t(86)=$ 13.48, $p<.001, d=1.065$, and incongruent versus neutral (mean difference $0 \mathrm{~ms}) t(86)=0.046, p=.96, d=0.00$. These results support our hypothesis that congruent color combinations delay disengagement significantly.

Response times Correct RTs were calculated from the onset of the search display to the button press. To test for a slowdown 
in RTs for congruent letter combinations as compared to incongruent letter combinations, a repeated-measures ANOVA was calculated with fixation circle position (left or center) as between-subject factor and color congruency (neutral, incongruent, or congruent) and letter congruency (incongruent or congruent) as within-subject factors. The main effects for color congruency, $F(2,86)=84.15, p<.001, \eta^{2}=.63$, and fixation circle position, $F(1,43)=7.57 p=.009, \eta^{2}=.15$, are significant. The main effect for letter congruency is not significant, $F(1,43)=1.08, p=.30, \eta^{2}=.023$. The interaction between color congruency and fixation circle position, $F(2$, $86)=7.11, p=.002, \eta^{2}=.053$, and color congruency and letter congruency, $F(2,86)=10.25, p<.001, \eta^{2}=.19$, are significant. However, the interaction between letter congruency and fixation circle position, $F(1,43)=3.37, p=.073, \eta^{2}=$ .071 , and color congruency, letter congruency, and fixation circle position, $F(2,86)=2.29, p=.11, \eta^{2}=.041$, is not significant. In post hoc t-tests, we analyzed the letter congruency effect separated for each fixation circle position. For the left fixation circle position, there was a significant slowdown in RTs for incongruent versus congruent letter combinations only for incongruent color combinations, $t(116.6)=3.75, p<$ $.001, d=0.33$, but not for congruent color combinations, $t(116.6)=1.24, p=.22, d=0.11$, or neutral color combinations, $t(116.6)=1.7, p=.092, d=0.15$. In contrast, the middle fixation circle position showed a significant slowdown in RTs for incongruent versus congruent letter combinations only for congruent color combinations, $t(116.6)=2.32, p=.022, d=$ 0.27 , but not for incongruent color combinations, $t(116.6)=$ $.16, p=.87, d=0.014$, or neutral color combinations, $t(116.6)=1.29, p=.2, d=0.17$. To sum up, only if the fixation circle position is in the middle, the letter congruency effect is comparable to Experiment 1 and 2.

In order to remain consistent with Experiment 3, we performed the above ANOVAs and t-tests again only for the adjacent circle of the left-most positioned fixation circle. We found that the color congruency effect is still prevalent (main effects: color congruency, $F(2,42)=8.6, p<.001, \eta^{2}=.29$, letter congruency, $F(1,21)=2.75 p=.11, \eta^{2}=.12$; interaction: $F(2,42)=0.9, p=.91, \eta^{2}=.004$.) with significant differences only for neutral $(631 \mathrm{~ms})$ versus congruent $(664 \mathrm{~ms})$ color combinations, $t(42)=3.27, p=.002, d=0.3$ and incongruent $(625 \mathrm{~ms})$ versus congruent $(664 \mathrm{~ms})$ color combinations, $t(42)=3.85, p<.001, d=0.38$. Contrary to Experiment 3, we found no significant letter congruency effect for congruent color combinations, $t(61.4)=0.75, p=.45, d=0.11$.

\section{References}

Becker, W., \& Jürgens, R. (1990). Human oblique saccades: Quantitative analysis of the relation between horizontal and vertical components.
Vision Research, 30(6), 893-920. https://doi.org/10.1016/00426989(90)90057-R

Belopolsky, A. V., Schreij, D., \& Theeuwes, J. (2010). What is top-down about contingent capture? Attention, Perception \& Psychophysics, 72(2), 326-341. https://doi.org/10.3758/APP.72.2.326

Biggs, A. T., \& Gibson, B. S. (2010). Competition between color salience and perceptual load during visual selection can be biased by topdown set. Attention, Perception \& Psychophysics, 72(1), 53-64. https://doi.org/10.3758/APP.72.1.53

Biggs, A. T., Kreager, R. D., Gibson, B. S., Villano, M., \& Crowell, C. R. (2012). Semantic and affective salience: The role of meaning and preference in attentional capture and disengagement. Journal of Experimental Psychology. Human Perception and Performance, 38(2), 531-541. https://doi.org/10.1037/a0027394

Blakely, D. P., Wright, T. J., Dehili, V. M., Boot, W. R., \& Brockmole, J. R. (2012). Characterizing the time course and nature of attentional disengagement effects. Vision Research, 56, 38-48. https://doi.org/ 10.1016/j.visres.2012.01.010

Boot, W. R., \& Brockmole, J. R. (2010). Irrelevant features at fixation modulate saccadic latency and direction in visual search. Visual Cognition, 18(4), 481-491. https://doi.org/10.1080/ 13506280903356780

Born, S., Kerzel, D., \& Theeuwes, J. (2011a). Evidence for a dissociation between the control of oculomotor capture and disengagement. Experimental Brain Research, 208(4), 621-631. https://doi.org/10. 1007/s00221-010-2510-1

Born, S., Kerzel, D., \& Theeuwes, J. (2011b). Evidence for a dissociation between the control of oculomotor capture and disengagement. Experimental Brain Research, 208(4), 621-631. https://doi.org/10. 1007/s00221-010-2510-1

Brockmole, J. R., \& Boot, W. R. (2009). Should I stay or should I go? Attentional disengagement from visually unique and unexpected items at fixation. Journal of Experimental Psychology. Human Perception and Performance, 35(3), 808-815. https://doi.org/10. 1037/a0013707

Egeth, H. E., \& Santee, J. L. (1981). Conceptual and perceptual components of interletter inhibition. Journal of Experimental Psychology: Human perception and performance, 7(3), 506. https://doi.org/10. 1037/0096-1523.7.3.506

Folk, C. L., Leber, A. B., \& Egeth, H. E. (2002). Made you blink! Contingent attentional capture produces a spatial blink. Perception \& Psychophysics, 64(5), 741-753. https://doi.org/10.3758/ BF03194741

Folk, C. L., Remington, R. W., \& Johnston, J. C. (1992). Involuntary covert orienting is contingent on attentional control settings. Journal of Experimental Psychology: Human perception and performance, 18(4), 1030. https://doi.org/10.1037/0096-1523.18.4. 1030

Gaspelin, N., Leonard, C. J., \& Luck, S. J. (2015). Direct Evidence for Active Suppression of Salient-but-Irrelevant Sensory Inputs. Psychological Science, 26(11), 1740-1750. https://doi.org/10. 1177/0956797615597913

Gaspelin, N., Ruthruff, E., \& Lien, M.-C. (2016). The problem of latent attentional capture: Easy visual search conceals capture by taskirrelevant abrupt onsets. Journal of Experimental Psychology. Human Perception and Performance, 42(8), 1104-1120. https:// doi.org/10.1037/xhp0000214

Heywood, S., \& Churcher, J. (1980). Structure of the visual array and saccadic latency: Implications for oculomotor control. The Quarterly Journal of Experimental Psychology, 32(2), 335-341. https://doi.org/10.1080/14640748008401169

James, K. H., \& Gauthier, I. (2006). Letter processing automatically recruits a sensory-motor brain network. Neuropsychologia, 44(14), 2937-2949. https://doi.org/10.1016/j.neuropsychologia.2006.06. 026 
Jamovi (Version 0.9): jamovi project (2018). Retrieved from https://www. jamovi.org

Laberge, D. (1973). Attention and the measurement of perceptual learning. Memory \& Cognition, 1(3), 268-276. https://doi.org/10.3758/ BF03198108

Leber, A. B., \& Egeth, H. E. (2006). It's under control: Top-down search strategies can override attentional capture. Psychonomic Bulletin \& Review, 13(1), 132-138. https://doi.org/10.3758/BF03193824

Sauter, M., Liesefeld, H. R., Zehetleitner, M., \& Müller, H. J. (2018). Region-based shielding of visual search from salient distractors: Target detection is impaired with same- but not differentdimension distractors. Attention, Perception \& Psychophysics, 80(3), 622-642. https://doi.org/10.3758/s13414-017-1477-4

Theeuwes, J. (1994). Stimulus-driven capture and attentional set: Selective search for color and visual abrupt onsets. Journal of Experimental Psychology: Human perception and performance, 20(4), 799. https://doi.org/10.1037/0096-1523.20.4.799

Theeuwes, J. (2004). Top-down search strategies cannot override attentional capture. Psychonomic Bulletin \& Review, 11(1), 65-70. https://doi.org/10.3758/BF03206462
Treisman, A. M., \& Gelade, G. (1980). A feature-integration theory of attention. Cognitive psychology, 12(1), 97-136. https://doi.org/10. 1016/0010-0285(80)90005-5

Wang, B., \& Theeuwes, J. (2018). Statistical regularities modulate attentional capture independent of search strategy. Attention, Perception \& Psychophysics, 80(7), 1763-1774. https://doi.org/10.3758/ s13414-018-1562-3

Wright, T. J., Boot, W. R., \& Brockmole, J. R. (2015a). Functional fixedness: The functional significance of delayed disengagement based on attention set. Journal of Experimental Psychology. Human Perception and Performance, 41(1), 17-21. https://doi.org/10. 1037/xhp0000016

Wright, T. J., Boot, W. R., \& Jones, J. L. (2015b). Exploring the breadth of the top-down representations that control attentional disengagement. Quarterly Journal of Experimental Psychology (2006), 68(5), 993-1006. https://doi.org/10.1080/17470218.2014.973888

Publisher's note Springer Nature remains neutral with regard to jurisdictional claims in published maps and institutional affiliations. 